\title{
Fetal MRI Mediastinal Shift Angle, Respiratory and Cardiovascular Pharmacological Support in Newborns With Congenital Diaphragmatic Hernia
}

llaria Amodeo

Fondazione IRCCS Ca' Granda Ospedale Maggiore Policlinico

Irene Borzani

Fondazione IRCCS Ca' Granda Ospedale Maggiore Policlinico

Giulia Corsani

Fondazione IRCCS Ca' Granda Ospedale Maggiore Policlinico

Nicola Pesenti

Fondazione IRCCS Ca' Granda Ospedale Maggiore Policlinico

Genny Raffaeli

Fondazione IRCCS Ca' Granda Ospedale Maggiore Policlinico

Francesco Macchini

Fondazione IRCCS Ca' Granda Ospedale Maggiore Policlinico

Valentina Condò

Fondazione IRCCS Ca' Granda Ospedale Maggiore Policlinico

Nicola Persico

Fondazione IRCCS Ca' Granda Ospedale Maggiore Policlinico

\section{Stefano Ghirardello}

Fondazione IRCCS Ca' Granda Ospedale Maggiore Policlinico

Mariarosa Colnaghi

Fondazione IRCCS Ca' Granda Ospedale Maggiore Policlinico

\section{Fabio Mosca}

Fondazione IRCCS Ca' Granda Ospedale Maggiore Policlinico

Giacomo Cavallaro ( $\square$ giacomo.cavallaro@policlinico.mi.it)

Fondazione IRCCS Ca' Granda Ospedale Maggiore Policlinico https://orcid.org/0000-0002-4921-1437

\section{Research Article}

Keywords: Fetal Magnetic Resonance Imaging, Mediastinal Shift Angle, Congenital Diaphragmatic Hernia, pulmonary vasodilators, inotropes

Posted Date: May 13th, 2021

DOI: https://doi.org/10.21203/rs.3.rs-497597/v1

License: (c) (i) This work is licensed under a Creative Commons Attribution 4.0 International License. Read Full License 
Version of Record: A version of this preprint was published at European Journal of Pediatrics on July 23rd, 2021. See the published version at https://doi.org/10.1007/s00431-021-04207-8. 


\section{Abstract}

Introduction. In newborns with congenital diaphragmatic hernia $(\mathrm{CDH})$, the mediastinal shift caused by the herniated organs negatively affects lung development. Assessment of the fetal magnetic resonance imaging (MRI) mediastinal shift angle (MSA) was shown to have an inverse correlation with the total fetal lung volume (TFLV), being associated with neonatal survival. However, a possible association with postnatal morbidity has never been investigated.

We hypothesize that the degree of the mediastinal shift could be associated with higher respiratory and cardiocirculatory impairment, requiring intensive treatments and extended hospitalization in survivors.

Method. We retrospectively consider a cohort of isolated, left-sided $\mathrm{CDH}$, for whom we calculated the MSA and the observed/expected (O/E) TFLV at fetal MRI. We performed a data collection regarding inotropic or vasoactive support, treatment with pulmonary vasodilators, mechanical ventilation, and length of stay. General linear models were performed.

Results. The MSA and O/E TFLV were inversely correlated (Pearson's coefficient $-0.65, p<0.001$ ) and deceased patients showed higher MSA values then survivors $(p=0.011)$. Among survivors, an increase in MSA was associated with longer pharmacological treatments (dobutamine: $p=0.016$; dopamine: $p=0.049$; hydrocortisone: $p$ $=0.003$; nitric oxide: $p=0.002$; sildenafil: $p=0.039$; milrinone: $p=0.039$; oxygen: $p=0.066$ ), and mechanical ventilation $(p=0.005)$, with an increasing trend in the length of hospitalization $(p=0.089)$.

Conclusions. The MSA indirectly reflects lung hypoplasia and is associated with the higher neonatal intensity of cares. Further studies are needed to consolidate the results.

Clinical Trial Registration: The study is an exploratory post-hoc analysis of the registered NeoAPACHE protocol at ClinicalTrials.gov with the identifier NCT04396028

\section{What Is Known}

- In congenital diaphragmatic hernia, the lung size, liver position, and defect side are the most common prenatal prognostic parameters used in clinical practice for morbidity and mortality prediction.

- Lung hypoplasia, strictly associated with lung size, is estimated by observed/expected lung to head ratio and observed/expected total fetal lung volume with prenatal ultrasound and fetal magnetic resonance imaging, respectively.

\section{What is new:}

- A new, faster, more straightforward, and less operator-dependent tool to assess CDH severity could be the mediastinal shift angle calculation with fetal magnetic resonance imaging.

- Postnatal clinical severity, considered as a postnatal cardiovascular and respiratory impairment that indirectly reflects lung hypoplasia, is associated with an increased mediastinal shift angle calculation.

\section{Introduction}

Congenital diaphragmatic hernia $(\mathrm{CDH})$ represents a rare congenital malformation affecting 1:3000 live births (1). Although advances in fetal and neonatal management should increase postnatal survival, overall mortality and 
morbidity remain high $(2,3)$. Prenatal diagnosis and risk stratification are relevant to assure the prompt referral to a

tertiary Center, proper counseling, and the correct selection of candidates for prenatal treatment $(4,5)$. However, due to the extreme variability of the disease, the prognostic prediction remains challenging (2).

Lung size, liver herniation, and defect side are the most common prenatal prognostic parameters used in clinical practice (6). In particular, lung size estimation is strictly associated with lung hypoplasia and postnatal survival chances (7). Lung size is most widely calculated as observed/expected (O/E) lung to head ratio (LHR) at prenatal ultrasound (US) (8). However, the assessment of the total fetal lung volume (TFLV) at fetal magnetic resonance imaging (MRI) was demonstrated to be more accurate than 2D US examination in predicting postnatal mortality and morbidity, representing an independent prognostic factor (5, 9-12). For outcome prediction, the absolute value of the TFLV is converted to a percentage of what is expected for a normal fetus of the same gestational age, based on normative data (O/E TFLV) (13).

However, since 3D lung volume reconstruction requires an experienced operator, a dedicated software, and could be time-consuming, the mediastinal shift angle (MSA) calculation has been recently proposed as a possible faster, easier, and less operator-dependent tool to assess $\mathrm{CDH}$ severity (14-16). Based on the assumption that lung volume is negatively influenced by the extent of the mediastinal shift caused by the amount of the herniated organs, the MSA was shown to have an inverse correlation with O/E LHR and TFLV and to be associated with neonatal survival in isolated left CDH (14-16).

However, the MSA assessment is quite novel, and few data from a single institution are available to date. Moreover, only neonatal mortality has been considered in those previous studies, while a possible association with neonatal clinical severity is lacking.

We hypothesize that the mediastinal shift could be associated with a higher need for intensive cares. In particular, the mechanical compression on the dislocated heart could determine a severe cardiocirculatory instability, requiring longer inotropic and vasoactive support and hospitalization. Similarly, an increased MSA is expected to determine a higher respiratory impairment, which could require more prolonged mechanical ventilation, oxygen supplementation, and treatment with pulmonary vasodilators.

\section{Methods}

This retrospective exploratory post-hoc analysis study was performed at Fondazione IRCCS Ca' Granda Ospedale Maggiore Policlinico, Milan, Italy, on a cohort of CDH patients over a 7-year period (2012 -2018).

\section{Subjects}

We considered all inborn $\mathrm{CDH}$ patients admitted to the Neonatal Intensive Care Unit (NICU). Among them, we selected all patients who had a fetal MRI. Patients were excluded if one or more of the following criteria were present: right-sided or bilateral $\mathrm{CDH}$, associated major malformations (with particular reference to thoracopulmonary and cardiac malformations), genetic anomalies or syndromes known to have an impact on postnatal survival, candidates to prenatal fetal endoscopic tracheal occlusion (FETO) who were enrolled in the TOTAL trial (www.totaltrial.eu). Therefore, the final study population was constituted by newborns with isolated, left-sided, and expectantly managed $\mathrm{CDH}$. All patients admitted to our NICU were managed according to the $\mathrm{CDH}$ EURO Consortium Consensus (17). In particular, hemodynamic status, the severity of CDH-associated pulmonary 
hypertension, and the need for pulmonary vasodilators and response to treatment were assessed through echocardiographic parameters (18-20).

\section{Data collection}

Data regarding prenatal history, clinical and surgical course were collected from the medical records of each patient. Data acquisition was anonymous. In particular, the following indicators of clinical severity were considered for outcomes analysis:

1. Inotropic and vasoactive support: days of treatment with dobutamine, dopamine, hydrocortisone.

2. Pulmonary vasodilators: days of treatment with oxygen, nitric oxide, sildenafil, milrinone;

3. Mechanical ventilation: days of high frequency oscillatory and/or conventional ventilation;

4. Length of stay: days from birth to NICU discharge.

\section{Fetal MRI examination}

Fetal MRIs were performed using a 1.5-Tesla system (Siemens Avanto) with a sixteen-channel body coil by a Pediatric Radiologist with consolidated experience in fetal MRI and prenatal assessment of CDH. Expecting mothers were supine or in left lateral decubitus, in the "feet first" position, and no sedative or intravascular contrast agent was administered. The MRI protocol for CDH consisted of the following sequences:

- Multiplanar localizer/true fast imaging with steady-state free precession (TRUFI), (TE 1.5, TR 3.6, slice thickness $4 \mathrm{~mm}$ )

- Three-plane Half-Fourier Acquisition Single-shot TSE (HASTE) T2-weighted according to the fetal head and body orientation, with FOV 380 and FOV 250 (TR 1000, TE 90, mm). No breath-hold was requested of the patient. The radiologist adjusted the field of view and the number of sections, and the image orientation for each fetus as required for optimal lung volume measurement. Sequences that were degraded by fetal motion were repeated with the same parameters.

- Three-plane Volumetric Interpolated Breath-hold Examination (VIBE) T1-weighted of the fetal body (TR: 6.7, TE: 2.3, slice thickness: $3 \mathrm{~mm}$ ) T1 Fast Low-Angle Shot fat-saturated (FLASH-FS) in the orthogonal transverse, coronal and sagittal planes

- Diffusion-Weighted Imaging (DWI) with b-value of 0,500, 1000.

Examination time was inferior to $25 \mathrm{~min}$ for all pregnant women. On the acquired sequences, the MSA and the lung volumes were measured for each patient.

\section{Mediastinal shift angle}

The MSA was measured by a Pediatric Radiologist with two years of experience in fetal MRI. The angle was measured on an axial HASTE image at the level of the four-chamber view of the fetal heart. A sagittal midline landmark line was drawn from the posterior face of the vertebral body to the mid of the posterior surface of the sternum, dividing the fetal thorax into two symmetric parts. A second landmark line was traced from the same vertebral point, representing the vertex of the angle, tangentially to the external wall of the right atrium (14-16). The software automatically calculated the corresponding MSA (figure 1). Three consecutive MSA measurements were performed for each patient, and the mean value was used for the analysis. 
The MSA was also independently assessed by a ten-year-experience Pediatric Radiologist on a sample of 10 random patients, and the concordance between the two operators was verified before performing the statistical analysis.

\section{Fetal lung volumes}

For each patient, lung volumes were calculated on the T2 HASTE sequences. We chose the axial, coronal, or sagittal plane corresponding to the best image quality, covering the whole thorax on a single acquisition and without motion-induced artifacts. On each section, left and right lung areas were independently determined using freehand region of interest (ROI) on Synapse 3D (FUJIFILM Medical Systems USA, Inc.). The main vessels of the pulmonary hila and mediastinal structures were excluded. The areas were automatically added and multiplied for the slice thickness and intergap by the software to obtain the entire volume of each lung. Left and right lung volumes were added to obtain the total fetal lung volume (TFLV) of each fetus (figure 2). The TFLV was then expressed as a percentage of the mean normal value expected for gestational age (O/E TFLV \%), as previously determined by Rypens et al. (13).

\section{Statistical analysis}

Continuous variables were reported as mean (standard deviation, SD) or median (interquartile range, IQR); categorical variables were presented as number and percentage. The concordance between the measurements of the two operators was assessed by calculating Lin's concordance correlation coefficient. For the comparison between groups, Student's T-Test, Mann-Whitney U Test, or Fisher Exact Test were performed, as appropriate.

The association between MRI measurements and the neonatal outcome was evaluated on patients who survived discharge since neonatal deaths occurring early after birth would have altered the analysis, preventing a reliable evaluation of the clinical course severity in these patients. General linear models were used to assess the association between the MSA with each of the studied outcomes. As a benchmark, the association with O/E TFLV was also investigated, and results were finally compared (13).

Statistical analysis was performed using IBM SPSSÒStatistics V26.0. A p-value of 0.05 or lower was considered to be statistically significant.

\section{Ethical considerations}

The present study was carried out in accordance with the principles of good clinical practice and the Helsinki Declaration, as well as the national legislative and administrative provisions in force. This study was approved by the local Ethics Committee (Milan Area 2, Italy), with approval number OSMAMI-04/05/2020-0015998-U. Due to the retrospective nature of the study, informed consent was waived by the Ethics Committee. The study is an exploratory post-hoc analysis of the registered NeoAPACHE protocol at ClinicalTrials.gov with the identifier NCT04396028.

\section{Results}

On 116 eligible patients, the assessment of the MSA and O/E TFLV was performed on 31 subjects (Figure 3 ). Demographics, clinical and radiological characteristics of the study population are reported in Table 1 (Table 1). Mean gestational age was $37.7 \pm 1.5$ weeks, mean birth weight was $2948 \pm 584 \mathrm{~g}$. Two patients died before surgical 
intervention, which was performed on 29 patients (93.5\%) with a patch required in 11 cases (37.9\%). Death occurred in 6 cases (19.4\%). Median length of stay was 42 (26-66) days.

MRI was performed on average at $28.7 \pm 3.3$ weeks of gestation. Therefore, the mean MSA was $33.7 \pm 6.7^{\circ}$, as calculated by the 2-year-experience radiologist, while the mean MSA was $32.7 \pm 8.7^{\circ}$, as measured by the 10 -yearexperience radiologist. Lin's concordance correlation coefficient was 0.993 (95\% Cl: 0.979 - 0.998). Mean O/E TFLV was $41.9 \pm 15.3 \%$.

According to the neonatal outcome, the study population was divided into two groups: deceased $(n=6)$ and survived $(n=25)$. The clinical and radiological characteristics of the two groups were compared (Table 2).

Deceased patients showed significantly higher MSA and lower O/E TFLV as compared to survivors (MSA: $38.5 \pm 3.7^{\circ}$ vs $32.6 \pm 6.8^{\circ}, p=0.011$; O/E TFLV: $30.2 \pm 10.9 \%$, vs $44.7 \pm 15 \%, p=0.034$ ) (Figure 4). The two parameters were inversely correlated (Pearson's correlation coefficient $-0.65, p<0.001$ ).

On survivors, regression analyses were performed to assess the relationship between the MSA and each studied outcome. An increase in the MSA was significantly associated with an increased duration of dobutamine $(p=$ $0.016)$, dopamine $(p=0.049)$ and hydrocortisone treatment $(p=0.003)$. An increased angle was also associated with an increased duration of mechanical ventilation $(p=0.005)$, nitric oxide $(p=0.002)$, sildenafil $(p=0.039)$, and milrinone treatment $(p=0.039)$, with a borderline statistical significance for oxygen supplementation $(p=0.066)$. The length of hospitalization also showed an increasing trend, even if not statistically significant $(p=0.089)($ Table 3, Panel A).

The association with O/E TFLV was then considered. An increase in O/E TFLV was associated with a reduced duration of dobutamine $(p=0.026)$, dopamine $(p=0.034)$ and hydrocortisone treatment $(p=0.003)$. An increased lung volume was also associated with a reduced duration of mechanical ventilation $(p=0.039)$, oxygen supplementation $(p=0.018)$, nitric oxide $(p=0.028)$ and sildenafil treatment $(p=0.016)$, but not milrinone $(p=$ $0.141)$. Length of hospitalization was shorter $(p=0.035)$ (Table 3 , Panel $B)$.

The main discordant finding was represented by milrinone treatment. As a supplementary analysis, we focused on the distribution of milrinone treatment days in survivors. As shown by the scatterplot, within the study population, two different subgroups could be identified: patients requiring at least one day of milrinone administration $(n=13)$ and those who received no treatment $(n=18)$ (Figure 5, Panel A). The mean MSA was significantly different between the treated and untreated group $\left(37.0 \pm 4.5^{\circ}\right.$ vs. $\left.31.3 \pm 7.0^{\circ}, p=0.010\right)$, while the $0 / E$ TFLV was not (36.6 $\pm 9.6 \%$ vs. $45.7 \pm 17.7 \%$, p 0.075) (Figure 5, Panel B).

\section{Discussion}

In isolated $\mathrm{CDH}$, the degree of lung hypoplasia represents the most important independent prognostic factor for neonatal survival (21-23).

Lung development is negatively influenced by the herniation of the abdominal organs through the diaphragmatic defect, displacing the mediastinal structures in the opposite direction. Therefore, the degree of the mediastinal shift is closely related to the total fetal lung volume and was found to be associated with neonatal survival in the recent literature. Our study confirmed an inverse correlation between the MSA and the O/E TFLV assessed at fetal MRI so that the MSA could be considered an indirect measure of lung hypoplasia. Indeed, the MSA and the O/E TFLV were 
significantly different between survivors and non-survivors. In particular, patients with poor outcomes were characterized by high MSA and low O/E TFLV values. As can be observed in the graphics, almost all high MSA cases had a poor prognosis, while most patients with low MSA survived to discharge.

The study showed an association between increased MSA and postnatal clinical severity. In particular, higher MSA values determined more significant postnatal cardiovascular and respiratory impairment, requiring longer inotropic and vasoactive pharmacological support with dobutamine, dopamine, and hydrocortisone, as well as more prolonged mechanical ventilation and use of pulmonary vasodilators such as nitric oxide, sildenafil, and milrinone. Moreover, patients receiving milrinone infusion were characterized by a more severe mediastinal shift. The same trend was also confirmed for oxygen supplementation, although with a borderline statistical significance.

As expected, the decrease in the O/E TFLV was associated with greater respiratory and cardiocirculatory impairment as well. However, the duration of milrinone administration was not influenced by the O/E TFLV.

Finally, high prenatal MSA and low O/E TFLV both influenced the overall length of hospitalization, although the former at a $10 \%$ significance level.

Our results suggest that the MSA reflects hernia severity and is strictly associated with increased mortality and a higher need for neonatal intensive cares. Increased MSA values could help identify a subgroup of patients at higher risk for prolonged NICU stay and second-line treatments, such as milrinone, better than the O/E TFLV alone.

Our findings are in accordance with the recent literature. On fetuses with left isolated CDH, Romiti and colleagues reported a mean MRI-MSA value of $43.7^{\circ}$ for deceased and $39.6^{\circ}$ for survivors. They identified a cut-off value of $39.1^{\circ}$ as having the highest discriminatory power to classify survival correctly (15). However, patients undergoing the FETO procedure in utero were not excluded from the study cohort, and the timing of the MSA assessment (preor post-FETO) was not reported. This aspect should be considered when interpreting these results, as the prenatal procedure could alter the MSA measurement by reducing the mediastinal shift and improving lung development.

However, in a previous paper, Savelli and colleagues evaluated the MSA on a cohort of expectantly managed, isolated, left-sided CDH. Both the mean MSA and TFLV were significantly different between survivors and nonsurvivors, and they identified a cut-off value of $38.2^{\circ}$ as having the highest discriminatory power to predict survival to discharge correctly (16). Our study had a similar sample size and mean MSA values. However, a comparison between lung volumes is not possible since Savelli and coll. referred to the absolute lung volume and not to the $\mathrm{O} / \mathrm{E}$ TFLV, which we consider more appropriate due to the important influence of gestational age on fetal lung development.

Nevertheless, the inverse correlation between MSA and lung volumes and the difference between survivors and nonsurvivors were confirmed. Finally, we decided not to perform a ROC curve analysis on our cohort due to the low sample size. For the same reason, we believe that the cut-off value identified by Savelli and coll. should be carefully used, as already recognized by the authors themselves, and should be confirmed on a larger CDH population.

Regarding neonatal cardiocirculatory and respiratory impairment, a comparison with the previous literature is not feasible. To our knowledge, the association between fetal MSA and neonatal clinical severity has never been investigated so far.

The presence of the hernia has a negative impact on the development of ipsilateral cardiac structures $(24,25)$. Compression of heart structures, abnormal cardiac axis, distorted fetal flow, increased pulmonary vascular 
resistance and decreased left ventricular pre-load contribute to a decreased cardiac output (26). Early ventricular dysfunction is an independent determinant for hernia severity and clinical outcome (26-28). In this regard, the O/E TFLV was shown to correlate with early postnatal left ventricular dysfunction, and both are associated with the need for extracorporeal membrane oxygenation (ECMO) in the neonatal period (3, 28-31). In light of these considerations, we can speculate that the MSA indirectly reflects the mechanical compression of the heart, resulting in a greater need for inotropic and vasoactive pharmacological support, such as dobutamine and dopamine. Patients with $\mathrm{CDH}$ have both left and right ventricular dysfunction, and a targeted selection of cardiovascular therapies is of key importance for optimal postnatal management (28). Right ventricular dysfunction requires pulmonary vasodilation to reduce afterload and lusitropic support to improve diastolic function. Instead, systemic vasodilation and inotropic support are required to reduce left ventricular afterload. Therefore, the phosphodiesterase 3 inhibitor milrinone is usually administered in the most severe forms of CDH since it combines all these pharmacological properties (32). Probably, our finding that the MSA was significantly higher in patients requiring milrinone is consistent with these considerations and deserves further analyses. Identifying infants at greater risk for cardiac dysfunction may allow for preventive management, and the MSA could help stratify those patients at a higher likelihood of severe cardiovascular impairment.

However, the relationship between the degree of the mediastinal shift and postnatal respiratory compromise is coherent with the impaired lung development caused by the mechanical compression of the herniated organs. Indeed, lung volume is a well-known marker of $\mathrm{CDH}$ severity. For example, lung volumes were found to be significantly lower in patients requiring a patch for hernia repair, who also received longer mechanical ventilation in the postoperative course (33). Moreover, low fetal MRI lung volumes have been associated with a postnatal need for oxygen supplementation and subsequent chronic lung disease development in previous studies $(29,31,33-36)$.

Finally, data regarding neonatal mortality are limited to a single-center experience based on a small number of patients. Therefore, our findings may contribute to the definition of the role of the MSA in predicting postnatal survival and the degree of cardiocirculatory and respiratory impairment.

The assessment of the MSA is simple, reproducible, and less time-consuming than lung volume 3D calculation. Moreover, it does not require an experienced operator nor dedicated software but can be easily performed on the most common MRI sequences. Therefore, the MSA assessment could represent an additional or alternative predictive tool, especially for less experienced operators working at peripheral institutions, to allow a prompt referral to a tertiary center for appropriate take in charge of the expecting mother (5). In these cases, the 3D fetal lung volume calculation could represent a second step performed by expert operators, helping in the optimal patient's pre- and postnatal management decision-making process. For example, it could more accurately identify cases that benefit from fetal balloon treatment or will likely require neonatal ECMO.

However, we also acknowledge some limitations in our study. First, the low sample size prevents us from making a proper statistical inference. Second, we did not consider several postnatal factors that could occur during the hospital course, influencing patient morbidity. Finally, we only performed a descriptive comparison of how MSA and O/E TFLV influenced the postnatal outcome.

To define which of the two measurements carries the highest prognostic capacity and consolidate the results, the MSA should be assessed and compared with lung volumetry on a larger CDH population.

As future directions, we also intend to perform the MSA assessment earlier during the pregnancy, ideally before 27 weeks of gestation for all patients, in order to evaluate if the MSA could correctly identify the candidates for the 
prenatal treatment and even predict the favorable response to the FETO procedure. We will also investigate a possible role of the MSA in predicting severe cardiorespiratory insufficiency and the need for neonatal ECMO.

\section{Conclusions}

The MSA indirectly reflects lung hypoplasia and patients with poor outcomes show a higher mediastinal shift. Among survivors, higher MSA values determine greater neonatal cardiovascular and respiratory impairment in terms of the longer need for inotropic and vasoactive support, pulmonary vasodilators, and mechanical ventilation, with an overall prolonged NICU hospitalization. Although respiratory impairment was expected due to altered lung development, the MSA might be more informative on cardiac dysfunction and hemodynamic status. Further studies are needed to consolidate the results and better define the prenatal MSA assessment role in the prognostic evaluation of left isolated $\mathrm{CDH}$.

\section{Declarations}

\section{Acknowledgments}

The authors would like to thank all the Neonatal ECMO Team Mangiagalli of the Fondazione IRCCS Ca' Granda Ospedale Maggiore Policlinico: nurses and neonatologists of the NICU, surgeons of the Department of Pediatric Surgery, anesthesiologists of the Pediatric Anesthesiology and Intensive Care Unit, nurses of the operating room.

\section{References}

1. Russo FM, De Coppi P, Allegaert K, Toelen J, Van Der Veeken L, Attilakos G, et al. Current and future antenatal management of isolated congenital diaphragmatic hernia. Seminars in Fetal and Neonatal Medicine. 2017;22(6):383-90.

2. Jani J, Nicolaides KH, Keller RL, Benachi A, Peralta CF, Favre R, et al. Observed to expected lung area to head circumference ratio in the prediction of survival in fetuses with isolated diaphragmatic hernia. Ultrasound Obstet Gynecol. 2007;30(1):67-71.

3. Russo FM, Eastwood MP, Keijzer R, Al-Maary J, Toelen J, Van Mieghem T, et al. Lung size and liver herniation predict need for extracorporeal membrane oxygenation but not pulmonary hypertension in isolated congenital diaphragmatic hernia: systematic review and meta-analysis. Ultrasound Obstet Gynecol. 2017;49(6):704-13.

4. Benachi A, Cordier A-G, Cannie M, Jani J. Advances in prenatal diagnosis of congenital diaphragmatic hernia. Seminars in Fetal and Neonatal Medicine. 2014;19(6):331-7.

5. Dütemeyer V, Cordier AG, Cannie MM, Bevilacqua E, Huynh V, Houfflin-Debarge V, et al. Prenatal prediction of postnatal survival in fetuses with congenital diaphragmatic hernia using MRI: lung volume measurement, signal intensity ratio, and effect of experience. J Matern Fetal Neonatal Med. 2020:1-9.

6. Claus F, Sandaite I, DeKoninck P, Moreno O, Cruz Martinez R, Van Mieghem T, et al. Prenatal anatomical imaging in fetuses with congenital diaphragmatic hernia. Fetal Diagn Ther. 2011;29(1):88-100.

7. Basurto D, Russo FM, Van der Veeken L, Van der Merwe J, Hooper S, Benachi A, et al. Prenatal diagnosis and management of congenital diaphragmatic hernia. Best Practice \& Research Clinical Obstetrics \& Gynaecology. 2019;58:93-106. 
8. Jani J, Nicolaides K, Keller R, Benachi A, Peralta C, Favre R, et al. Observed to expected lung area to head circumference ratio in the prediction of survival in fetuses with isolated diaphragmatic hernia. Ultrasound in obstetrics \& gynecology. 2007;30(1):67-71.

9. Jani J, Cannie M, Done E, Van Mieghem T, Van Schoubroeck D, Gucciardo L, et al. Relationship between lung area at ultrasound examination and lung volume assessment with magnetic resonance imaging in isolated congenital diaphragmatic hernia. Ultrasound Obstet Gynecol. 2007;30(6):855-60.

10. Bebbington M, Victoria T, Danzer E, Moldenhauer J, Khalek N, Johnson M, et al. Comparison of ultrasound and magnetic resonance imaging parameters in predicting survival in isolated left-sided congenital diaphragmatic hernia. Ultrasound Obstet Gynecol. 2014;43(6):670-4.

11. Jani J, Cannie M, Sonigo P, Robert Y, Moreno O, Benachi A, et al. Value of prenatal magnetic resonance imaging in the prediction of postnatal outcome in fetuses with diaphragmatic hernia. Ultrasound Obstet Gynecol. 2008;32(6):793-9.

12. Victoria T, Bebbington MW, Danzer E, Flake AW, Johnson MP, Dinan D, et al. Use of magnetic resonance imaging in prenatal prognosis of the fetus with isolated left congenital diaphragmatic hernia. Prenat Diagn. 2012;32(8):715-23.

13. Rypens F, Metens T, Rocourt N, Sonigo P, Brunelle F, Quere MP, et al. Fetal lung volume: estimation at MR imaging-initial results. Radiology. 2001;219(1):236-41.

14. Romiti A, Viggiano M, Conforti A, Valfré L, Ravà L, Ciofi Degli Atti M, et al. Ultrasonographic assessment of mediastinal shift angle (MSA) in isolated left congenital diaphragmatic hernia for the prediction of postnatal survival. J Matern Fetal Neonatal Med. 2020;33(8):1330-5.

15. Romiti A, Viggiano M, Savelli S, Salvi S, Vicario R, Vassallo C, et al. Comparison of mediastinal shift angles obtained with ultrasound and magnetic resonance imaging in fetuses with isolated left sided congenital diaphragmatic hernia. J Matern Fetal Neonatal Med. 2020:1-6.

16. Savelli S, Bascetta S, Carducci C, Carnevale E, Caforio L, Romiti A, et al. Fetal MRI assessment of mediastinal shift angle in isolated left congenital diaphragmatic hernia: A new postnatal survival predictive tool? Prenat Diagn. 2020;40(1):136-41.

17. Snoek KG, Reiss IK, Greenough A, Capolupo I, Urlesberger B, Wessel L, et al. Standardized postnatal management of infants with congenital diaphragmatic hernia in Europe: the CDH EURO consortium consensus-2015 update. Neonatology. 2016;110(1):66-74.

18. Sanchez Mejia AA, Rodgers NJ. Evaluation and Monitoring of Pulmonary Hypertension in Neonates With Congenital Diaphragmatic Hernia. Curr Treat Options Cardiovasc Med. 2019;21(2):11.

19. Gupta VS, Harting MT. Congenital diaphragmatic hernia-associated pulmonary hypertension. Semin Perinatol. 2020;44(1):151167.

20. Puligandla PS, Skarsgard ED, Offringa M, Adatia I, Baird R, Bailey M, et al. Diagnosis and management of congenital diaphragmatic hernia: a clinical practice guideline. CMAJ. 2018;190(4):E103-E12.

21. Hedrick HL, Danzer E, Merchant AM, Bebbington MW, Zhao H, Flake AW, et al. Liver position and lung-to-head ratio for prediction of extracorporeal membrane oxygenation and survival in isolated left congenital diaphragmatic hernia. Am J Obstet Gynecol. 2007;197(4):422.e1-4.

22. Werner NL, Coughlin M, Kunisaki SM, HirschI R, Ladino-Torres M, Berman D, et al. Prenatal and postnatal markers of severity in congenital diaphragmatic hernia have similar prognostic ability. Prenat Diagn. 2016;36(2):107-11. 
23. Schopper MA, Walkup LL, Tkach JA, Higano NS, Lim FY, Haberman B, et al. Evaluation of Neonatal Lung Volume Growth by Pulmonary Magnetic Resonance Imaging in Patients with Congenital Diaphragmatic Hernia. J Pediatr. 2017;188:96-102.e1.

24. DeKoninck P, D'hooge J, Van Mieghem T, Richter J, Deprest J. Speckle tracking echocardiography in fetuses diagnosed with congenital diaphragmatic hernia. Prenat Diagn. 2014;34(13):1262-7.

25. Byrne FA, Keller RL, Meadows J, Miniati D, Brook MM, Silverman NH, et al. Severe left diaphragmatic hernia limits size of fetal left heart more than does right diaphragmatic hernia. Ultrasound Obstet Gynecol. 2015;46(6):688-94.

26. Altit G, Bhombal S, Van Meurs K, Tacy TA. Diminished Cardiac Performance and Left Ventricular Dimensions in Neonates with Congenital Diaphragmatic Hernia. Pediatr Cardiol. 2018;39(5):993-1000.

27. Patel N, Lally PA, Kipfmueller F, Massolo AC, Luco M, Van Meurs KP, et al. Ventricular Dysfunction Is a Critical Determinant of Mortality in Congenital Diaphragmatic Hernia. Am J Respir Crit Care Med. 2019;200(12):152230.

28. Patel N, Massolo AC, Paria A, Stenhouse EJ, Hunter L, Finlay E, et al. Early Postnatal Ventricular Dysfunction Is Associated with Disease Severity in Patients with Congenital Diaphragmatic Hernia. J Pediatr. 2018;203:4007.e1.

29. Walleyo A, Debus A, Kehl S, Weiss C, Schönberg SO, Schaible T, et al. Periodic MRI lung volume assessment in fetuses with congenital diaphragmatic hernia: prediction of survival, need for ECMO, and development of chronic lung disease. AJR Am J Roentgenol. 2013;201(2):419-26.

30. Büsing KA, Kilian AK, Schaible T, Endler C, Schaffelder R, Neff KW. MR relative fetal lung volume in congenital diaphragmatic hernia: survival and need for extracorporeal membrane oxygenation. Radiology. 2008;248(1):240-6.

31. Schaible T, Büsing KA, Felix JF, Hop WC, Zahn K, Wessel L, et al. Prediction of chronic lung disease, survival and need for ECMO therapy in infants with congenital diaphragmatic hernia: additional value of fetal MRI measurements? Eur J Radiol. 2012;81(5):1076-82.

32. Patel N. Use of milrinone to treat cardiac dysfunction in infants with pulmonary hypertension secondary to congenital diaphragmatic hernia: a review of six patients. Neonatology. 2012;102(2):130-6.

33. Petroze RT, Caminsky NG, Trebichavsky J, Bouchard S, Le-Nguyen A, Laberge JM, et al. Prenatal prediction of survival in congenital diaphragmatic hernia: An audit of postnatal outcomes. J Pediatr Surg. 2019;54(5):92531.

34. Debus A, Hagelstein C, Kilian AK, Weiss C, Schönberg SO, Schaible T, et al. Fetal lung volume in congenital diaphragmatic hernia: association of prenatal MR imaging findings with postnatal chronic lung disease. Radiology. 2013;266(3):887-95.

35. Zamora IJ, Olutoye OO, Cass DL, Fallon SC, Lazar DA, Cassady Cl, et al. Prenatal MRI fetal lung volumes and percent liver herniation predict pulmonary morbidity in congenital diaphragmatic hernia (CDH). J Pediatr Surg. 2014;49(5):688-93.

36. Tsuda H, Kotani T, Miura M, Ito Y, Hirako S, Nakano T, et al. Observed-to-expected MRI fetal lung volume can predict long-term lung morbidity in infants with congenital diaphragmatic hernia. J Matern Fetal Neonatal Med. 2017;30(13):1509-13.

\section{Tables}

Page 12/20 
Table 1. Demographics, clinical and radiological characteristics of the study population $(n=31)$.

\section{Table 1. Demographics, clinical and radiological characteristics of the study population \\ $\mathrm{CDH}(\mathrm{n}=31)$}

Prenatal Data

Initial O/E LHR\% - mean ( \pm SD)

$43.8(14.6)$

Final O/E LHR\% - mean ( \pm SD)

$44.4(15.6)$

Liver Up - n (\%)

$12(38.7)$

Grading - n (\%)

Severe

Moderate

Mild

$14(45.2)$

Neonatal Data

$\mathrm{GA}$ (weeks) - mean $( \pm S D)$

$37.7(1.5)$

Weight $(g)-$ mean $( \pm S D)$

$2948(584)$

Males - n (\%)

APGAR $1^{\circ} \mathrm{min}$ - median (IQR)

$6(4.75-7)$

APGAR $5^{\circ} \mathrm{min}$ - median (IQR)

$8(8-9)$

Operated - n (\%)

$29(93.5)$

Day of surgery - median (IQR)

$3(2-4)$

Diaphragmatic patch (on operated) - n (\%)

$11(37.9)$

Length of stay (days) - median (IQR)

$42(26-66)$

Deaths - n (\%)

$6(19.4)$

Imaging Data

GA at MRI (weeks) - mean $( \pm S D)$

$28.7(3.3)$

O/E TFLV (\%) - mean ( \pm SD)

$\operatorname{MSA}\left({ }^{\circ}\right)-$ mean $( \pm S D)$

$33.7(6.7)$

GA: gestational age; IQR: interquartile range; MRI: magnetic resonance imaging; MSA: mediastinal shift angle; O/E LHR\%: observed/expected lung to head ratio; O/E TFLV\%: observed/expected total fetal lung volume; SD: standard deviation.

Table 2. Comparison of the characteristics between deceased $(n=6)$ and survived $(n=25)$ patients. 
Table 2. Comparison of the characteristics between deceased and survived patients

Deceased $(n=6) \quad$ Survived $(n=25) \quad p$ value

\section{Prenatal Data}

Initial O/E LHR\% - mean ( \pm SD)

$33.8(9.2)$

$46.5(14.8)$

$0.022^{*}$

Final O/E LHR\% - mean ( \pm SD)

$31.8(10.0)$

$46.9(15.4)$

$0.023^{*}$

Liver Up - n (\%)

$6(100)$

$6(24)$

$<0.001^{\wedge}$

Grading - n (\%)

Severe

1 (3.8)

$0(0)$

$0.006^{\wedge}$

Moderate

$5(83.0)$

$11(44)$

Mild

$0(0)$

$14(56)$

\section{Neonatal Data}

GA (weeks) - mean ( \pm SD)

$36.3(2.4)$

$38(1)$

$0.147 *$

Birth weight $(\mathrm{g})$ - mean $( \pm \mathrm{SD})$

2498 (511)

3056 (556)

$0.045^{\star}$

APGAR $1^{\circ} \mathrm{min}$ median (IQR)

$5(4-5.25)$

$7(5.25-7)$

$0.030^{\circ}$

APGAR $5^{\circ}$ min median (IQR)

$8(7-8.25)$

$8(8-9)$

$0.278^{\circ}$

Operated $-\mathrm{n}(\%)$

4 (66.7)

$25(100)$

$0.032^{\wedge}$

Day of surgery

$2.5(1.25-4.5)$

$3(2-4)$

$0.379^{\circ}$

Diaphragmatic patch (on operated) - $\mathrm{n}(\%)$

$3(75)$

8 (32)

$0.139^{\wedge}$

Mechanical ventilation (days) - median (IQR)

$3(0.75-10)$

$10(9-21.5)$

$0.005^{\circ}$

Oxygen (days) - median (IQR)

$5(1.75-10)$

$14(4.5-24.5)$

$0.073^{\circ}$

Nitric Oxyde (days) - median (IQR)

$5(1.5-9.5)$

$8(0-17)$

$0.527^{\circ}$

Sildenafil (days) - median (IQR)

$5(1.5-8.25)$

$22(0-40)$

$0.213^{\circ}$

Milrinone (days) - median (IQR)

$3(0-7)$

$0(0-7.5)$

$0.469^{\circ}$

Dobutamine - median (IQR)

$4(1.75-6.5)$

$3(0-9)$

$0.860^{\circ}$

Dopamine - median (IQR)

$3(1-7.25)$

$4(2-7.5)$

$0.598^{\circ}$

Hydrocortisone - median (IQR)

$5(1.75-8.5)$

$6(0-8.5)$

$0.880^{\circ}$

Length of stay (days) - median (IQR)

$5(1.75-10)$

$43(36.5-70)$

$<0.001^{\circ}$

\section{Imaging Data}

GA at MRI (weeks) - mean $( \pm S D)$

$27(2.1)$

$29.2(3.4)$

$0.072^{\star}$

O/E TFLV (\%) - mean ( \pm SD)

$30.2(10.9)$

44.7 (15)

0.034 *

$\operatorname{MSA}\left({ }^{\circ}\right)$ - mean $( \pm S D)$

$38.5(3.7)$

$32.6(6.8)$

$0.011^{*}$ 
GA: gestational age; IQR: interquartile range; MRI: magnetic resonance imaging; MSA: mediastinal shift angle; O/E LHR\%: observed/expected lung to head ratio; O/E TFLV\%: observed/expected total fetal lung volume; SD: standard deviation.

Fisher Exact Test $\left({ }^{\wedge}\right)$, Mann Whitney U-Test $\left({ }^{\circ}\right)$, or Student's T-Test $\left(^{\star}\right)$ were performed as indicated.

Table 3. Association between mediastinal shift angle (MSA), observed/expected total fetal lung volume (O/E TFLV), and intensive cares duration in CDH survivors. Panel A: Linear regression analysis between MSA and neonatal outcome; Panel B: Linear regression analysis between O/E TFLV and neonatal outcome.

Table 3. Association between mediastinal shift angle (MSA), observed/expected total fetal lung volume (O/E TFLV), and intensive cares (days) in CDH survivors.

Neonatal intensive cares in survivors

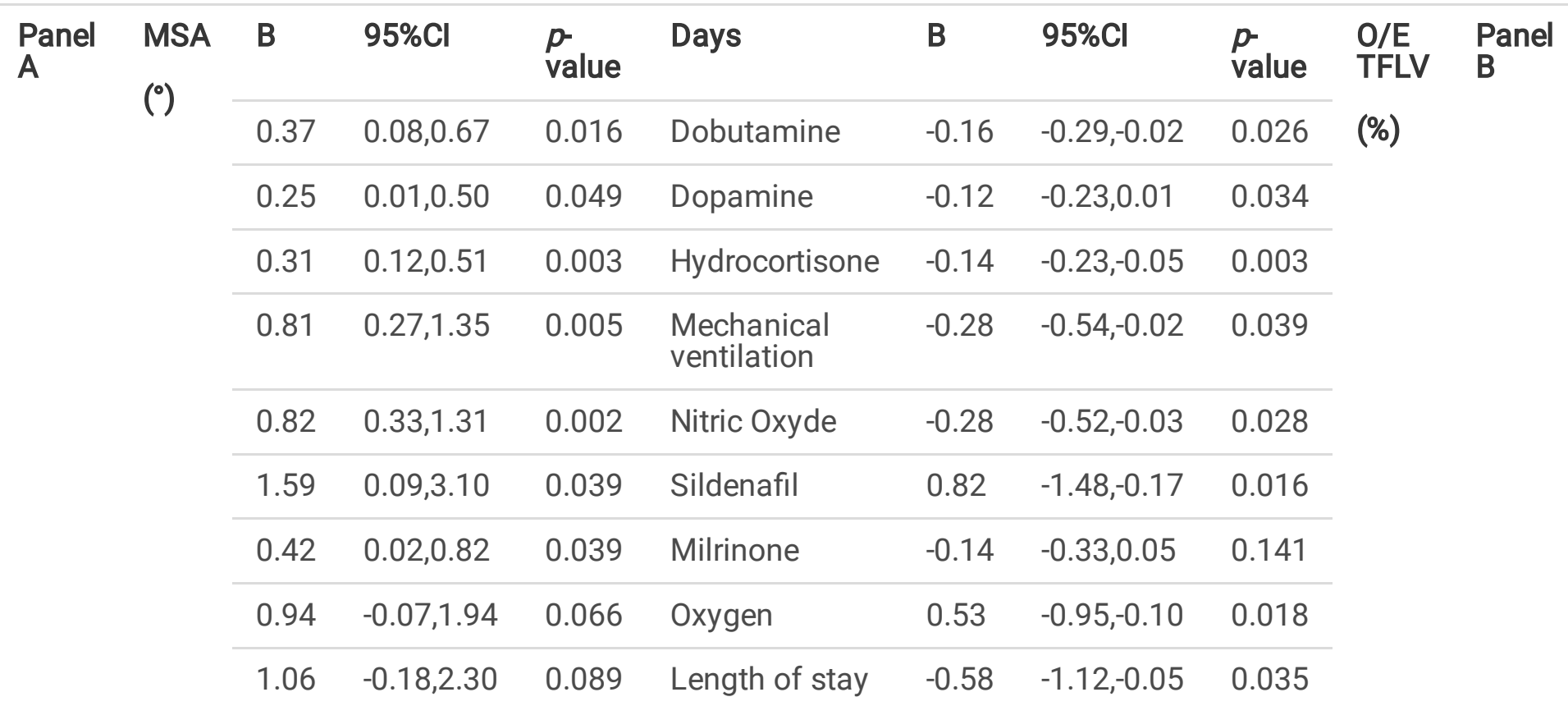

MSA: mediastinal shift angle; O/E TFLV: observed/expected total fetal lung volume.

\section{Figures}




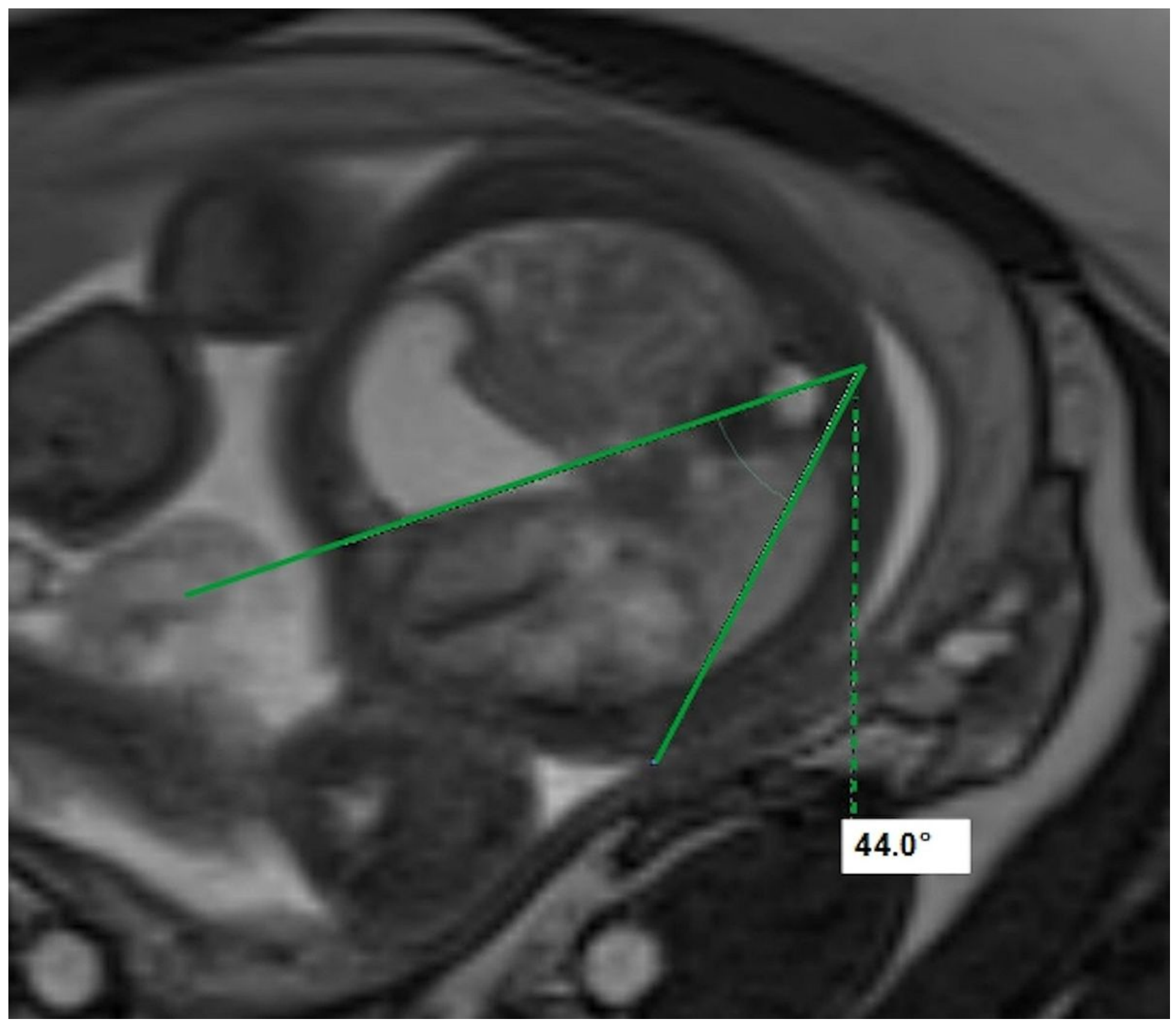

\section{Figure 1}

Axial HASTE T2-weighted image at the level of the four-chamber view of the fetal heart. The two lines delimit the angle of the mediastinal shift. 


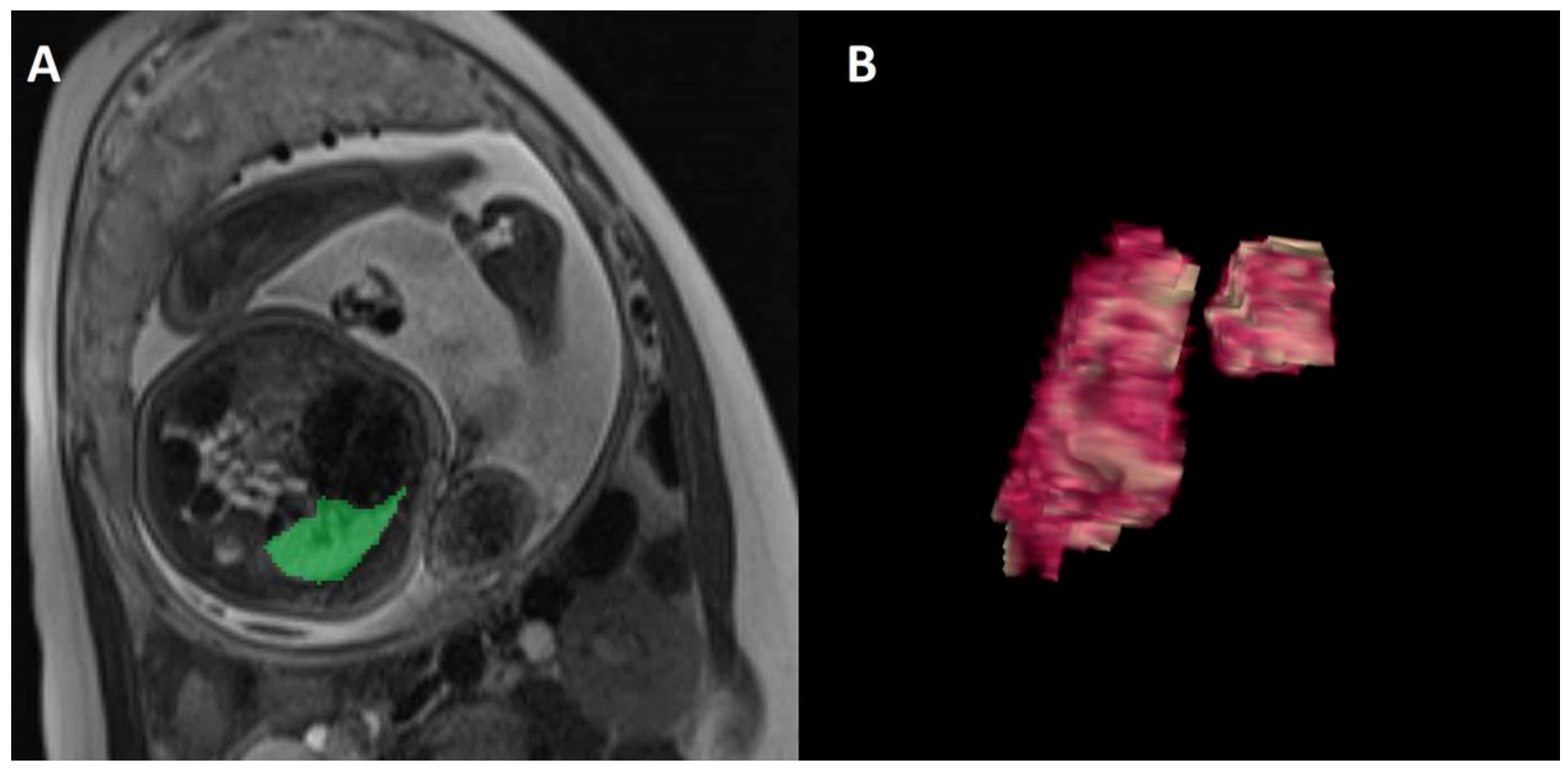

Figure 2

Calculation of the fetal lung volume. A: Axial HASTE T2-weighted image of the chest and free hand lung segmentation; B: 3D lung reconstruction of the total fetal lung volume. 


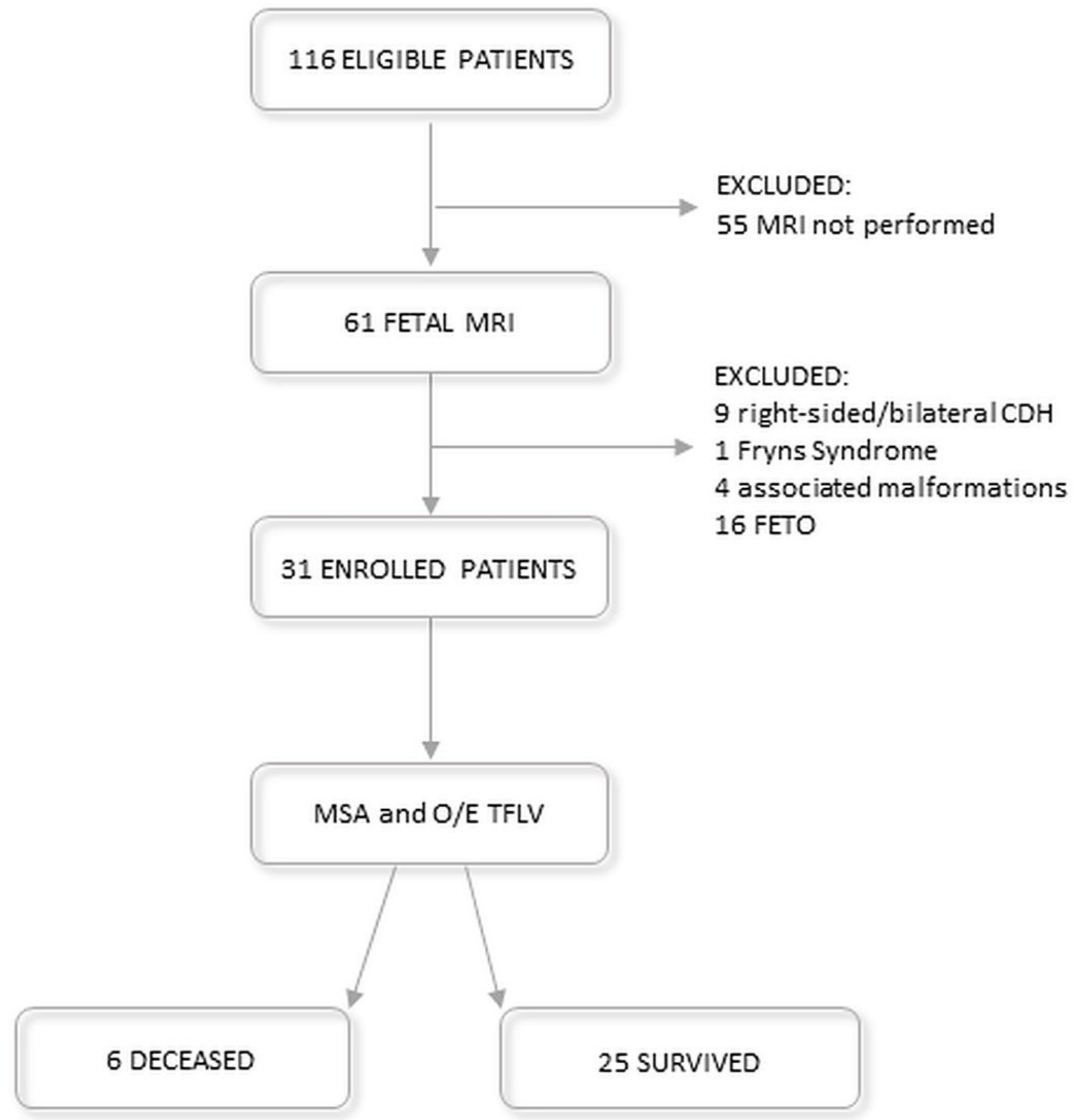

Figure 3

Study flowchart. 
A

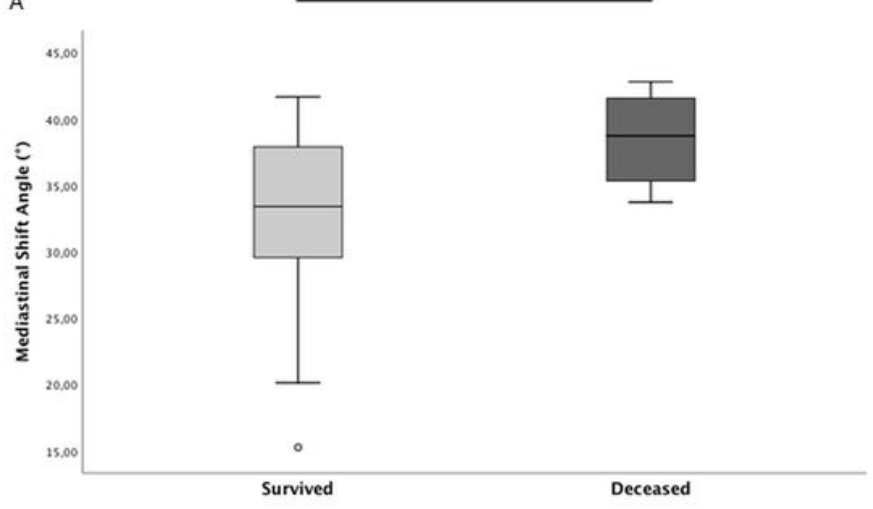

B

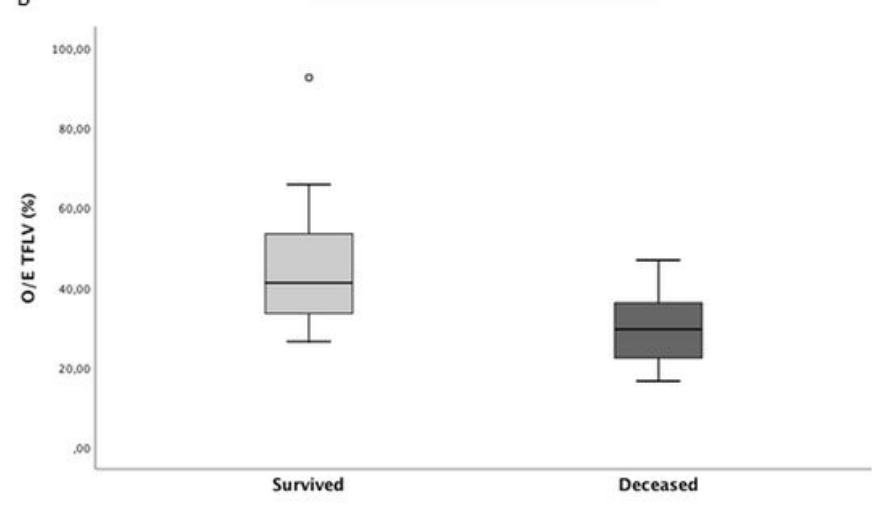

Figure 4

Boxplot showing the distribution of the Mediastinal shift single (MSA) and observed/expected total fetal lung volume $\left(\mathrm{O} / \mathrm{E}\right.$ TFLV) in deceased $(n=6)$ and survived $(n=25)$ patients. $A: M S A\left({ }^{\circ}\right), p=0.011 ; B: 0 / E$ TFLV $(\%), p=$ 0.034 .
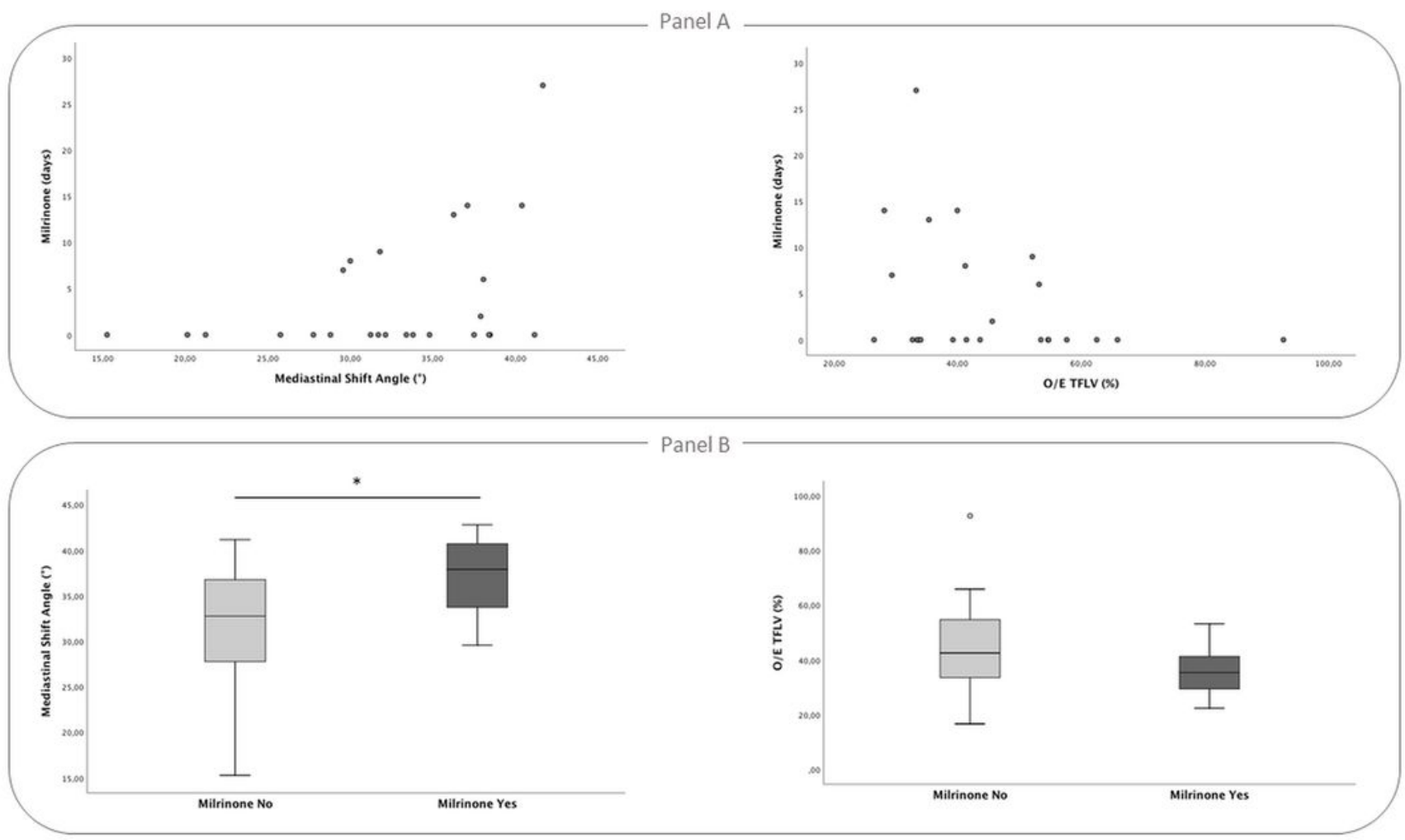

Figure 5

Milrinone treatment in survivors. Panel A: Scatterplot showing the distribution of milrinone treatment duration in relation to the mediastinal shift angle (MSA) and observed/expected total fetal lung volume (O/E TFLV) in 
survivors. Panel B: Boxplot showing the distribution of the mediastinal shift angle (MSA) and observed/expected total fetal lung volume $(O / E T F L V)$ values in patients receiving $(n=13)$ and not receiving $(n=18)$ milrinone treatment. MSA $\left({ }^{\circ}\right), p=0.010 ; 0 / E \operatorname{TFLV}(\%), p=0.075$. 